\title{
Energy and architecture - An overview
}

\author{
G. $\operatorname{Sonetti}(*)$ \\ Architect, MSc. The University of Nottingham, University Park, NG7 2RD, Nottingham, UK
}

\begin{abstract}
Summary. - This paper aims to provide a short overview on the complex aspects and growing concern about energy in architecture by gradually zooming into it, starting from a macro-scale analysis of building contribution in the total EU energy consumption, related policies, user behaviour's impacts and vernacular architecture techniques; then looking at the meso scale of building energy performance during its use, dynamic simulations of heat transfer and insights from a whole life cycle analysis of the energy involved during construction and disposal phases; finally, at the building element micro-scale, describing local heat transfer and human thermal comfort measurements. Conclusions gather recommendations and further scenarios where different stakeholders and techniques can play their part for a wiser and more sustainable energy use, and a better built environment for us and those to come.
\end{abstract}

\section{1. - Introduction}

If a system can be modelled as composed by 3 parameters, and each parameter can assume " $n$ " number of states, then we know that the total combination of possible configurations is $3^{n}$. What happens with buildings is that even if the number of parameters that can be observed regarding energy issues could be relatively low, the " $n$ " always prompts very large, and - to make things harder - to weigh key parameters in a hi-

$\left(^{*}\right)$ E-mail: giulia.sonetti@gmail.com 
erarchy scale and in a common frame valid for every case, is not a trivial matter. In addition, since achieving thermo-hygrometric comfort deals also with economic, security, safety and environmental issues, the sustainability challenge for an architecture design team or a policy maker nowadays becomes even tougher. Choosing a configuration over another requires a strong simplification effort to translate the multitude of physical interactions into "edible" data. A building is characterized by several thermodynamics equations (air movement, radiation exchange, noise transmission, moisture flow, electrical power flow, degree of insulation, etc.) each one of which may interact by a non-linear law with the others, to not mention the repercussion that each singular variation can have on the total energy consumption, or the transportation and maintenance costs, or the disposal scenario: " $n$ " variations correspond to incredibly high cascade results.

This paper will cover essential issues at the macro, meso and micro scale of this complex topic about energy and built environment, stressing the outlines for further improvements in energy saving techniques and user environmental awareness.

\section{2. - Energy issue at macro scale: the built environment}

$2 \cdot 1$. Quick facts on building energy consumption trends. - We are continuously bombarded by frightened data on climate change emergency, $\mathrm{CO}_{2}$ emissions and energy source depletion, reserves' scarcity and building contributing to this worsening situation. Statistics exposed afterwards are not the $n$-th threatening messages and apocalyptic scenarios running to the usual final audience switching off, but constitute an important preface to understand the border conditions of the problem and calibrate weights of different energy issues in built environment.

Energy consumption of buildings in developed countries comprises $20-40 \%$ of the total energy use [1] and is above industry and transport figures in EU and USA. Future projections indicate that in 2030, buildings will be responsible for $35.6 \%$ of primary energy use in the world and continue to maintain their importance. Causes of this can be found in the high rate of growth in population, the enhancement of building services and the very high standard of living, together with the rise in time spent inside a building. In addition, predictions indicate a massive growth in energy consumption in EU within the next 15 years, increasing approximately around 50\%. According to building energy consumption trends, Spain energy consumption rate is above the North American (1.9\%) and European data (1.5\%) and much above the UK figure. This can be the result of increased building sector business, wider availability of building services and in particular large use of HVAC (heating, ventilation and air conditioning) systems. Emerging economies, too, will intensify needs for education, health and other services, together with the consequential energy consumption [2].

In the residential sector, weather, architectural design, energy systems and economic level of the occupants are important indicators for energy consumption. By and large, small flats need less energy as there is less conditioned and transfer area, and also less occupation, and dwellings in developed countries use more energy than those in emerging economies due to the installation of new appliances (air conditioners, computers, etc.). 
In USA, dwellings consume $22 \%$ of the total final energy use, compared with $26 \%$ in the EU. The UK figure is $28 \%$, well above the Spanish $15 \%$, and this is mainly due to a more severe climate and to the predominance of independent houses over blocks in the house typology. In non-domestic buildings, the type of use and activities make a huge impact on the quality and quantity of energy services needed. HVAC is the main end use with a rate close to 50\%. Lighting follows with $15 \%$ and appliances with $10 \%$. Building typology is crucial for energy consultancy and housing stock assessment, since it influences both the distribution and the intensity of energy use at building and district level. Within the commercial sector, office buildings are, together with retail, those with the biggest consumption and $\mathrm{CO}_{2}$ emissions.

$\mathbf{2} \cdot 2$. Building energy policies. - EU last FP7 calls emphasize on energy rating to inform and stimulate subsequent building renovation activities [3]. About $85 \%$ of the European buildings are older than 20 years; $60 \%$ are older than 40 years and $30 \%$ are pre-war buildings. Most of them are not equipped with advanced building management and control systems. It is estimated that about $50 \%$ of these buildings will be renovated in the next 20 years opening the potential to improve their energy performance [2]. Local administrations can seriously reduce emissions only by increasing the environmental awareness of citizens and stakeholders and helping them to take action. The new European Directive on the energy performance of buildings [4] requires each country to implement policies to improve the efficiency of buildings, until new buildings have almost zero energy consumption by 2020. Thus, local administrations could accomplish a lot by persuading citizens to retrofit their houses. The Italian national tax relief policy has led to energy saving retrofits, but primarily to those that require less financial commitment, such as window replacement. These retrofits result in lower energy savings compared to those on opaque building surfaces but are a lot cheaper and so easier to apply. Therefore, to achieve the European Union targets for 2020, it will be essential encouraging private initiative together with government intervention, promoting of energy efficiency, new technologies for energy production, limiting energy consumption and raising social awareness on the rational use of energy.

23. Passive design and hybrid approach. - Building energy efficiency can be locally improved by implementing either active or passive energy efficient strategies. Improvements to heating, ventilation and air conditioning (HVAC) systems, electrical lighting, etc. can be categorized as active strategies, whereas improvements to building envelope elements can be classified under passive strategies. Recent years have seen a renewed interest in environmental-friendly passive building energy efficiency strategies, because they are being envisioned as a viable solution to the problems of energy crisis and environmental pollution. Energy savings of $31.4 \%$ and peak load savings of $36.8 \%$ from the base case were recorded for high-rise apartments in the hot and humid climate of Hong Kong just by implementing passive energy efficient strategies [5]. In a different study [6], the thermal and heat transfer performance of a building envelope in the subtropical climatic conditions of Hong Kong saved as much as $35 \%$ and $47 \%$ of total and peak cooling demands, respectively. 
Advanced and sustainable materials research for building envelope applications has seen significant progress in recent years, but a proper design inspired by past examples is a worthwhile endeavour and a scientific guidance as valid as high-tech solutions. Vernacular architectures, recovered for example by Assan Fathy and then other architects, are those that are built by people, thus influenced by tradition and culture. They survived up to our era because they have been gleaned through a long period of trial and error and the ingenuity of local builders who possess specific knowledge about their place on the planet, so they form a tangible proof of effectiveness of climate-specific passive building technologies to modern buildings [7]. In a world that is seeing the rapid dissemination of mass-produced homes and buildings designed by teams of architects and engineers sometimes thousands of miles away from the building site, there is a threat that vernacular traditions that help define the cultural make-up of a people and a place will be lost. The challenge to preserve these valuable techniques and cultures will be to package this knowledge in such a way that vernacular traditions become widely known to modern designers and builders, including information about not only the energy performance but also about cost, material availability and cultural traditions [8].

Conclusion of a comparative study [9] aiming to explore the influence of incorporating ICT and modern techniques in buildings in hot climates through the perspective of passive and active building performances, shows that dependence of individual active features to solve one or some climatic design strategies brings energy savings that range from just $8 \%$ up to $40 \%$, according on the combination used. Passive design strategies that used low-sophistication devices achieved between $20 \%$ to $60 \%$ energy savings, but some of these strategies might not be applicable to all cases (for example, depending on south-facing orientations only). This makes them unreliable for constant savings in all situations. However, combining active features and correct passive design strategies gives consistent savings of around 50-55\% for most cases when compared to a conventional situation. Such sensibly planned buildings offer flexibility and convenience that exclusively passive buildings cannot give, such as rapid temporary changes, options to open individual windows or operate specific blinds. A successful smart building and smart city cannot be just a collection of smart active features: it needs to be a product of a design process that incorporates intelligence in all its stages, including the schematic early ones, evaluating socioeconomic impacts of each choice while taking advantage of technological innovations and local resources.

$\mathbf{2}$ 4. RES integration. - Renewable energy source integration within new and existing buildings is also a significant approach to reduce resource consumption. In the European Directive 31 definition of the concept of a "zero" or "near zero" energy building (ZEBs or near ZEBs), the energy use in new constructions must be compensated by the production of energy from renewable sources. The energy balance will be zero when the energy demand equals the renewable-energy generation, both on-site supply options available within the building's footprint (solar photovoltaics, small and medium wind turbines, solar thermal, CHP, heat pumps) and off-site site supply (hydro electricity, biomass, wind and solar farm) [10]. 
Currently, three systems have been receiving wide concern, including solar-based energy systems, ground source-based energy systems and day-lighting systems. Recently, Marszal [11] determined the life cycle cost of the on-site and off-site renewable energy supply systems: from the private economy perspective and with the current technologies' cost and energy price, in 4 out of 5 on-site RES options investment in energy efficiency is a more cost-effective decision than investment in renewable-energy technologies. The off-site RES options have a reverse life cycle cost trend, and for all systems the combination of less demining energy frame and high renewable-energy generation is the most cost-optimal path towards net-zero building.

25. User behaviour in the domestic building. - Evidence from behavioural economics and psychology outlines the current approach to enabling people, at home and at work, to reduce their energy consumption and reduce their bills in the process. Behaviourally based changes that reduce emissions have indeed major advantages. First, the benefits can be very fast, unlike major infrastructure changes that can take years, or even decades, and a $1 \%$ gain today is worth more than a $1 \%$ gain tomorrow. Second, they can be highly cost-effective. Third, they can provide savings and other benefits directly to citizens. A detailed survey held by Lopes [12] also suggested acting on user awareness of stand-by power consumption (that represents the $8-10 \%$ of total consumption) and in general on a closer association between electricity production and its economic burden, since energy cost is always the main priority even for residents enthusiastic about energy savings. Studies in the United States, the Netherlands and the UK [13] have estimated that 26$36 \%$ of in-home energy use is due to residents behaviour. To promote energy-conscious behaviour effectively throughout the population requires a better understanding of the interface between people and the equipment they use. In general, further attention should be given to assessing information transfer methods that combine the frequently updated appliance-specific feedback interface (to increase motivation to save energy) with feasible energy-saving tips at the points-of-use (to increase knowledge about energysaving methods). For example, energy-saving tips might be displayed on a drop-down menu whilst the user is new to the Energy Consumption Indicators (ECI), and then hidden by the user once he/she knows about the tips or has modified certain energy use practises. This feature would play a secondary but potentially important role to that part of the display that indicates energy consumption.

\section{3. - Energy issue at the meso scale: the building}

According to the definition in the EPBD Recast Annex 3, "The energy performance of a building shall be expressed in a transparent manner and shall include an energy performance indicator and a numeric indicator of primary energy use, based on primary energy factors per energy carrier, which may be based on national or regional annual weighted averages or a specific value for on-site production". This methodology uses primary energy as the metric for building energy performance to "indicate the quality of a building in energy use" [14]. The most commonly used EP indicator for many building 
types is energy use intensities (EUI), i.e. $\mathrm{kWh} / \mathrm{m}^{2}$. Building energy performance is mainly determined by six factors: climate, building envelope, building services and energy systems, building operation and maintenance, occupants' activities and behaviour and indoor environmental quality provided, as summarized in IEA Annex 53 project. The energy performance assessment approaches in the building sector can be classified into two major categories, namely performance-based and feature-specific approaches [15]. Using the performance-based approach, assessment results are obtained by comparing the performance indicators (e.g. EUI or $\mathrm{CO}_{2}$ emission) against established benchmarks.

Dynamic simulation programs have been accepted as powerful tools for analyzing building energy performance, where required inputs should be firstly collected and then be fed into a so-called simulation engine to describe detailed mathematic models. Typical inputs may include four groups of parameters, i.e. weather conditions, building description, system description and component description. The weather conditions generally include the dry and wet bulb temperature of outdoor air, solar radiation intensity, wind speed, etc. Building description data mainly include location, design and construction data, thermal zones, internal heat gain, infiltration and usage profiles, etc. For system description, system types and sizes, control schedules, as well as outdoor air requirements are needed. The inputs for component description are mainly addressed HVAC components, including the equipment types and sizes, performance characteristic, load assignments and auxiliary equipment. Simplified and steady state methods are generally acceptable to simulate the performance of systems and equipment for a diagnostic purpose or at sketch phases of building design.

3`1. The building energy budget. - The building energy budget may be written, symbolically, as this equation presented by Taesler [16] (see fig. 1):

$$
Q_{\mathrm{C}}+Q_{\mathrm{G}}+Q_{\mathrm{F}}+Q_{1}-Q_{\mathrm{P}}-Q_{\mathrm{E}}-Q_{\mathrm{S}} \pm \Delta Q=Q_{\mathrm{N}},
$$

where: $Q_{\mathrm{C}}=$ heat loss by conduction to the outside air, $Q_{\mathrm{G}}=$ heat loss by conduction to the ground, $Q_{\mathrm{F}}=$ heat loss by mechanical ventilation, $Q_{1}=$ heat loss by air leakage (infiltration), $Q_{\mathrm{P}}=$ heat gain from occupants, $Q_{\mathrm{E}}=$ heat gain from electric appliances, $Q_{\mathrm{S}}=$ heat gain from solar irradiation, $\Delta Q=$ heat storage in the building structure, $Q_{\mathrm{N}}=$ net energy input needed.

Equation (1) applies to all time scales, i.e., for calculating short-term (hourly, daily) heating/cooling demands, monthly and annual totals or long-term mean values. For the longer time scales, however, the storage term $\Delta Q$ may be neglected. Atmospheric conditions directly influence the terms $Q_{\mathrm{C}}, Q_{\mathrm{F}}, Q_{1}$ and $Q_{\mathrm{S}}$ on an hourly basis. $Q_{\mathrm{E}}$ is partially affected in response to day lighting conditions. $Q_{\mathrm{G}}$ depends on soil temperature at different depths. This term, however, is usually quite small and may be ignored or just roughly estimated. The terms $Q_{\mathrm{C}}$ and $Q_{\mathrm{F}}$ are, to a good approximation, proportional to the indoor-outdoor temperature difference $(\Delta T)$ and may thus be calculated from heating degree-hours or -days. $Q_{1}$ depends on $\Delta T$ in combination with wind speed and wind direction and $Q_{\mathrm{S}}$ depends on cloudiness and turbidity in addition to the position 


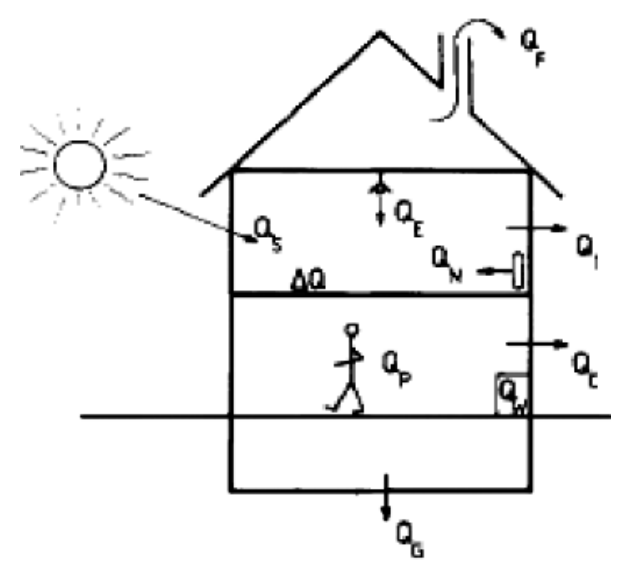

Fig. 1. - The building energy budget.

of the Sun in the sky. In order to calculate these terms it is necessary to account for the location and exposure of the building. Wind is strongly influenced by the local urban surroundings, and solar irradiation on building surfaces is even more strongly influenced by site-specific conditions. Temperature may also vary significantly in response to local and urban conditions. In addition, energy is needed for hot water production $\left(Q_{\mathrm{W}}\right)$. One of the designer's first decisions is to set an energy use goal for the building. This is a crucial programming level design choice that will effect both the process of how the building is designed and the final design product. With appropriate computerised engineering tools, professionals would be able to evaluate various energy saving options to be translated to "in the pocket" savings for the public and private read acceptability and feasibility.

3.2. LCA for the whole building life cycle energy consumption. - A wide review by Khasreen [17] estimated that the use phase in conventional buildings represents approximately $80 \%$ to $90 \%$ of the life-cycle energy use, while $10 \%$ to $20 \%$ is consumed by the material extraction and production and less than 1\% through end-of-life treatments. However, by the development of energy-efficient buildings and the use of less-polluting energy sources, the contribution of the material production and end-of-life phases is expected to dramatically increase in the future.

Life Cycle Assessment (LCA) is a useful methodology for evaluating the environmental and energy loads of processes during their whole life cycle, encompassing the extraction and processing of raw materials, manufacturing, transportation and distribution, use, reuse, maintenance, recycling and final disposal. Obviously, allocating the correct amount of energy used, for example, a brick fabrication, transportation, cutting and positioning on site, and forecast the amount of energy required for its transportation to recycle plants and its new shaping, is not an easy task [18]. The long life time of a 
building makes impossible an exact evaluation on a 70 years base; borders conditions change sensibly after a substitution or a loss or a misuse in a building element, there is very little standardization in techniques and then each building is unique and is designed as such. However, sustainability assessment is a combination of different tools, and the exact content of the assessment is planned on the basis of the project in question. Keeping in mind that "all models are wrong, and someone is useful", LCA can be very helpful in specifying energy impacts in the very early stage of a design process, gaining insight of the most energy-absorbing steps and understanding, from cradle to grave, how each choice can affect the whole building performance. LCA apart, systematic and effective methods for the management of performance characteristics are a prerequisite: information content from different objects (components, technical systems, buildings, spaces) needs to be known, in order to develop methods of producing the information. The challenge is to focus in the correct way, and thus to produce and process valid performance information for different stakeholders. The development potential of total building performance is in fitting experimental and calculative methods together in diagnostic methods, and in defining the criteria of a sustainable built environment metabolism [19].

3.3. Heat transfer at the building envelope level. - Coming back to the ultimate reason why buildings use energy, the final purpose of every building-plant system is to maintain comfortable living and working conditions in it. The temperature outdoors swings above and below comfortable temperatures, and the building envelope has to provide a thermal barrier between them. The amount of energy that flows across this barrier determines how much energy must be expended in heating or cooling the building to maintain comfort; therefore the efficiency and the correct use of the interface, including walls, roof, foundation and openings, plays an important role. The energy contained in a building is increased, for instance, by sunshine and by the supply of electricity and fuel for heating and other appliances [20]. Energy flows "in transit" through the control volume of a building by primary heat transport modes like:

- Conduction (heat flow on a molecular scale. Medium at rest or moving);

- Convection (heat conveyed as internal thermal energy of mass that is displaced by mean or turbulent motion);

- Radiation (heat transfer by electromagnetic waves such as infrared or visible light).

In buildings, heat is also transported by the following mechanisms, which basically belong to the convective mode:

- Transfer of latent heat by transport of water or water vapour

- Thermal energy associated with the air replaced in a building by ventilation or by air leakage (infiltration).

- Thermal energy associated with fresh and used domestic water and combustion air (including flue gases), and fluids feeding Heat Pumps. 
The transport of energy in the above list is limited to energy in the form of sensible or latent heat. A change of sensible heat is characterized by a change of temperature while a change of latent heat is associated with some mass altering its phase, while transport of energy in forms other than heat is usually not considered.

\section{4. - Energy issue at the micro scale - the building element}

According to the second law of thermodynamics, in a closed system heat transfer is only possible in the direction from a higher temperature to a lower one. The heat loss through an envelope should therefore be proportional to the difference $T_{\text {inside }}-T_{\text {outside }}$, or to a positive power of it for small differences. For a simple formula, a linear dependence on temperature difference is sufficient. Accepting further that heat loss grows linearly with surface area A, one finds

$$
\dot{Q}=A U\left(T_{i}-T_{e}\right)
$$

The constant of proportionality, $U$, is the Overall Heat Transfer Coefficient in $W /\left(\mathrm{m}^{2} \mathrm{~K}\right)$.

Equation (2) suggests three ways to reduce heat loss: 1) reduce the set-point for the indoor temperature during the heating season; 2) improve the insulation of the envelope and so the overall heat transfer coefficient $U ; 3)$ reduce the surface area without changing the enclosed volume.

The cumulated amount of lost heat is the time integral of the instantaneous heat flow,

$$
Q=\int \dot{Q} d t
$$

The quantity of heat, $Q$, is measured in $\mathrm{J}$ (Joule). In the construction sector, it is often converted into $\mathrm{kWh}$ (kW-hours). The fuel consumed for heating is roughly proportional to the difference between $Q$ and the sum of the internal heat gains from Sun, occupants, lights, equipment, and so forth. Therefore, the time-average of the temperature difference $\Delta T_{\text {bal }}=T_{\text {balance }}-T_{\text {outside }}$ during the heating season is of importance for estimating heating cost. Here, $T_{\text {balance }}$ is that outside temperature at which no heating is required to maintain a prescribed inside temperature at given internal heat gains [21]. For simplified calculation purpose, the thermal performance of buildings can be compared on the basis of degree-days $\left(\mathrm{DD}_{\mathrm{h}}\right)$, which correspond for a geographical site between given dates to a time integral of $\Delta T_{\text {bal }}$ in which only positive values of the difference are counted.

4.1. Human comfort and HVAC systems. - The human body is essentially a constanttemperature device. Heat is continuously produced by bodily processes and dissipated in an automatically regulated manner to maintain the body temperature at its correct level despite variations in ambient conditions. In terms of physiology, the body is in a state of thermal equilibrium with its environment when it loses heat at exactly the same rate as it gains heat. Mathematically, the relationship between the body's heat production 
and all its other heat gains and losses is [22]

$$
M=E \pm R \pm C \pm S
$$

where: $M=$ metabolic rate, $E=$ rate of heat loss by evaporation, respiration, and elimination, $R=$ radiation rate, $C=$ conduction and convection rate, $S=$ body heat storage rate.

The body always produces heat, so the metabolic rate $(M)$ is always positive, varying with the degree of exertion.

If environmental conditions are such that the combined heat loss from radiation, conduction, convection, and evaporation is less than the body's rate of heat production, the excess heat must be stored in body tissue. But body heat storage $(S)$ is always small because the body has a limited thermal storage capacity. Therefore, as its interior becomes warmer, the body reacts to correct the situation by increasing blood flow to the skin surface and increasing perspiration. As a result, body heat loss is increased, thereby maintaining the desired body temperature and the balance expressed by eq. (3). The converse condition - where heat loss is greater than body heat production - causes a reversal of the above process and, if necessary, shivering. This increased activity raises the metabolic rate. The comfort of a given individual is affected by many variables (see table I). Health, age, activity, clothing, gender, food and acclimatization are all determining factors of the comfort conditions for any particular person. In addition

TABle I. - Factors influencing the heat balance equation. Source: John Blankenbaker, "Ventilating Systems for Hot Industries," Heating/Piping/Air Conditioning, Vol. 54, No. 2, February 1982, p. 61. (Reproduced from the original: Industrial Ventilation. American Conference of Governmental Industrial Hygienists, Committee on Industrial Ventilation, Lansing, Michigan, 1976, p. 3-2.)

\begin{tabular}{|l|l|l|}
\hline Factor & Environment & Human \\
\hline Metabolism $(M)$ & Little effect & Activity \\
& & Weight \\
& & Surface area \\
& & Age \\
& & Sex \\
\hline Evaporation $(E)$ & Wet-bulb temperature & Ability to produce sweat \\
& Dry-bulb temperature & Clothing \\
& Velocity & Surface area \\
\hline Radiation $(R)$ & Temperature difference & Surface area \\
& between bodies & Clothing \\
& Emissivity of surfaces & \\
\hline Convection $(C)$ & Dry-bulb temperature & Clothing \\
& Velocity & Mean body surface temperature \\
& & Surface area \\
\hline
\end{tabular}




\section{LNES2012}

to its thermal climate, the air quality of each indoor environment affects the sense of comfort [23]. A new control strategy for automatic or customizable control of personalized radiant heating/cooling, maybe by including the human body as sensor in the control loop, can save a big amount of energy while maintaining thermal comfort of the individual building occupant.

\section{5. - Conclusions}

Relationships between energy and architecture, design, energy needs, disposal scenarios and user behaviour are in truth extremely complex. Buildings must be considered as holistic, dynamic systems, with many factors influencing different impact categories and needing complex evaluation tools to take into account complex relationship between energy, internal comfort and environmental impacts. Issues discussed above are evidence of a multidisciplinary approach on building design, in order to integrated planning with contributions from engineer, physics, chemistry and environmental and medical science.

A conscientious choice about environmental, societal and economic impacts in the design phase will be a cost-less energy saving along the whole life cycle. In the end, an insistent efficiency program in the building sector would prevent a large portion of the demand for new power stations and give more time to develop environmentally friendly energy-supply methods. Not only are constitute opportunities to apply today's efficiency knowledge, but advanced technologies - drawing on basic research by scientists and engineers in solid-state physics, thermodynamics, turbulent flows, and nanotechnology - could allow us to dramatically reduce today's building energy demand. To accelerate those developments, governmental and manufacturing R\&D efforts need to be significantly expanded, which would have the added gain of boosting educational and business opportunities for the next generation of leaders in the building-efficiency field.

\section{REFERENCES}

[1] EC-European Commission, New EU energy plan - More security, less pollution. Available at: http://ec.europa.eu/news/energy/070110, 2007.

[2] PÉrez-Lombard L., Ortiz J. et al., Energy and Buildings, 43 (2011) 255.

[3] EC-European Commission, Environment - Climate change - Emission Trading System. Available at: http://ec.europa.eu/environment/climat/emission/index en.htm, 2009.

[4] Directive 2010/31/UE, "The energy performance of buildings", Official Journal of the European Union, 2010.

[5] Shi L. and Chew M. Y. L., Ren. Sust. Energy Rev., 16 (2012) 192.

[6] Sadineni S. B., Madala S. et al., Ren. Sust. Energy Rev., 15 (2011) 3617.

[7] Brown G. Z., in Sun, wind, and light: architectural design strategies (Wiley, NY) 1985.

[8] Zhai Z. and Previtali J. M., Energy and Buildings, 42 (2010) 357.

[9] Ochoa C. E. and Capeluto I. G., Building and Environment, 43 (2008) 1829.

[10] Dall'O' G., Galante A. et al., Sustainable Cities and Society, 4 (2012) 12.

[11] Marszal A. J. and Heiselberg P. et al., Renewable Energy, 44 (2012) 154.

[12] Lopes L. and Hokoi S. et al., Energy and Buildings, 37 (2005) 698.

[13] Mansouri-Azar I., Newborough M. and Probert D., Applied Energy, 54 (1996) 211. 
[14] ECBCS, Total Energy Use in Buildings: Analysis and Evaluation Methods, Annex No. 53, 2009, p. 34.

[15] Wang S., Yan C. et al., Energy and Buildings, 55 (2012) 873.

[16] TAESLER R., Energy and Buildings, 16 (1991) 599.

[17] Khasreen M. M., Banfill P. F. G. et al., Sustainability, 1 (2009) 674.

[18] NeRI P., in Verso la valutazione ambientale degli edifici: life cycle assessment a supporto della progettazione eco-sostenibile (Alinea, Firenze) 2008.

[19] Giampietro M., Mayumi K. et al., Energy, 34 (2009) 313.

[20] Çengel Y. A., in Heat Transfer: A Practical Approach (McGraw-Hill, NY) 2003.

[21] American Society of Heating, ASHRAE Handbook: HVAC applications (ASHRAE, Atlanta) 2007.

[22] Fanger P. O., in Thermal comfort: Analysis and applications in environmental engineering (Danish Technical Press, Copenhagen) (1970).

[23] Blankenbaker J., Heating/Piping/Air Conditioning, 54 (1982) 60. 